\title{
http://dx.doi.org/10.35381/racji.v5i1.636
}

\section{Problemática de tenencia compartida a partir del interés superior de los niños, niñas y adolescentes}

\section{Problems of shared ownership based on the best interests of children and adolescents}

Daniela Araceli Quevedo-Sacoto

daniela.quevedo@ucacue.edu.ec

Universidad Católica de Cuenca, Cuenca

Ecuador

https://orcid.org/0000-0001-6459-8556

Juan Carlos Erazo-Álvarez

jcerazo@ucacue.edu.ec

Universidad Católica de Cuenca, Cuenca

Ecuador

https://orcid.org/0000-0001-6480-2270

Ernesto Marcelo Robalino-Peña

erobalino@ucacue.edu.ec

Universidad Católica de Cuenca, Cuenca

Ecuador

https://orcid.org/0000-0002-0753-7842

Cecilia Ivonne Narváez-Zurita

inarvaez@ucacue.edu.ec

Universidad Católica de Cuenca, Cuenca

Ecuador

https://orcid.org/0000-0002-7437-9880

Recibido: 13 de noviembre de 2019

Aprobado: 17 de diciembre de 2019

\section{RESUMEN}

En este artículo se efectúa un estudio del régimen de la tenencia compartida a partir del principio de igualdad y el interés superior de los niños, niñas y adolescentes, reconocidos en la Constitución de la República del Ecuador, así como, en el Código de la Niñez y Adolescencia, entre otros, instrumentos internacionales de derechos 
humanos. Se plantea como objetivo analizar la importancia del ejercicio conjunto de la patria potestad para el desarrollo integral de los menores, por cuanto en ciertas ocasiones después de una separación los padres se preocupan únicamente de sus intereses personales, dejando de lado las exigencias de sus hijos. Se empleó la metodología cualitativa, lo cual permite concluir la necesidad de equiparar las obligaciones de los progenitores para con sus hijos, a través de un modelo de ejercicio conjunto de la patria potestad que les otorgue las mismas obligaciones y derechos de educación y crianza.

Descriptores: Tenencia compartida; Código de la Niñez y Adolescencia; custodia; guarda; patria potestad.

\begin{abstract}
In this article, a study of the regime of shared tenure is carried out based on the principle of equality and the best interests of children and adolescents, recognized in the Constitution of the Republic of Ecuador, as well as in the Code of Children and Adolescence, among others, international human rights instruments. The objective is to analyze the importance of the joint exercise of parental authority for the integral development of minors, because on certain occasions after a separation, parents care only about their personal interests, leaving aside the demands of their children. The qualitative methodology was used, which allows to conclude the need to equate the obligations of the parents towards their children, through a model of joint exercise of parental authority that grants them the same obligations and rights of education and parenting.
\end{abstract}

Descriptors: Shared tenure; Childhood and Adolescence Code; custody; guard; custody.

\title{
INTRODUCCIÓN
}

Desde la Constitución del 2008 se ha dado una importante evolución del derecho constitucional, esto ha permitido que el estado ecuatoriano atraviese por tres modelos; el Clásico de Derecho, el Estado Social de Derecho y en la actualidad el Estado Constitucional de Derechos y Justicia, siendo este último el que nos interesa al contemplar la aplicación directa e inmediata de las normas constitucionales con estricto apego a los derechos humanos. De donde resulta que, la Constitución de la República 
será la encargada de garantizar los derechos de la familia para que exista un desarrollo integral de los niños, niñas y adolescentes y puedan tener una vida digna y un desarrollo emocional adecuado, frente a una tenencia de los menores monoparental, en la que, la guarda y la custodia es dada a uno de los progenitores que generalmente es la madre mientras que al padre se le reconoce únicamente el derecho contemplado en el régimen de visitas (Zabala, 2014).

De esta manera, el análisis del tema de la tenencia compartida pretende que los progenitores después de un divorcio ejerzan los deberes y derechos sobre los hijos basados en el interés superior de los menores y que de esta manera puedan tener la oportunidad de gozar tanto de su padre como de su madre en igual tiempo y calidad (Cajamarca, 2016), pero al mismo tiempo, el entendimiento de esta institución, coloca el problema de investigación, en lo referente a la importancia de la continuación del ejercicio equitativo de la patria potestad en favor de ambos progenitores, para el desarrollo emocional de los hijos, ya que en ciertas ocasiones después de un divorcio o la separación de los padres, estos solo se preocupan de sus intereses personales, despreocupándose de las necesidades de los menores y de la aplicación de la ley en cuanto a la tenencia de los hijos.

Así pues, estando claro que los hijos son lo más importante dentro del núcleo familiar, se pretende que después de un divorcio o una separación, los mismos tengan la oportunidad de tener lo más parecido a una familia y de conservar la figura clara de una madre y un padre, que sean capaces de compartir con sus hijos el mismo tiempo y calidad de vida en garantía de su derecho al buen vivir, con la finalidad de que no sufran los golpes de la separación de los progenitores y a futuro generen traumas por el hecho de haber tenido padres irresponsables. De ahí que, en relación con las implicaciones que esta figura crea, se considera que la tenencia compartida es muy importante para el desarrollo de los hijos, por lo que, habrá que dejar a un lado los problemas de los padres para poner por encima la felicidad de los hijos, y que a futuro sean hombres y mujeres felices y de bien (Tamayo Vásquez \& Pino Loza, 2019). 
Bajo estos argumentos, se plantea como problema de la presente investigación la siguiente pregunta: ¿Cuáles son las características que debe tener una reforma de la institución de la tenencia compartida regulada en el Código de la Niñez y Adolescencia del Ecuador a partir del interés superior de los niños, niñas y adolescentes? En función a la problemática expuesta el objetivo será proteger los derechos constitucionales de los niños, niñas y adolescentes y equiparar los derechos y obligaciones de los progenitores protegiendo de esta manera a la familia como núcleo fundamental de la sociedad.

\section{DESARROLLO}

\section{Concepto jurídico de tenencia compartida en la doctrina}

La tenencia compartida es un concepto jurídico que puede ser definido como aquella relación en la que los progenitores separados y no convivientes, colaboran entre sí y acuerdan en mantener un vínculo de apoyo recíproco, activamente comprometido con la vigilancia y crianza de sus hijos menores. En este sentido, Morales y Castillo (2011) afirman que la tenencia compartida es un derecho que mantiene cada: "(..) hombre y cada mujer que es padre de continuar siéndolo enteramente y no de forma parcial como ocurre con frecuencia cuando se concede la custodia exclusiva de los hijos a uno de los padres después de la separación o divorcio" (p. 57).

De forma similar, Badaraco (2018) define la tenencia compartida como: “(...) la situación legal por medio de la cual en caso de divorcio o separación matrimonial ambos padres ejercen simultáneamente la custodia legal de sus hijos menores de edad de acuerdo a las mismas condiciones y derechos sobre ellos" (p. 5). De donde se infiere que, esta institución es una modalidad de tenencia, que supone la continuación del ejercicio equitativo, frecuente y complementario de la patria potestad en su totalidad en favor de ambos progenitores, que incluye también la custodia estricta de los hijos, por cuanto, solamente manteniendo un trato respetuoso y fluido con sus padres, aunque estén 
divorciados, se puede conservar vigente la corresponsabilidad parental como su fundamento y fin.

Se trata, por tanto, de un quebrantamiento de la modalidad clásica de custodia monoparental, parcial y exclusiva, inicialmente regulada como la única forma efectiva de proteger el interés del menor, al colocarle bajo el cuidado de uno de los progenitores en contraposición del otro al que se le concedía únicamente ciertos derechos de vistas, función y comunicación, en una clara restricción de sus derechos paterno filiares con el impacto negativo que esto generaba en el menor. Sin embargo, esta idea irá progresando en el ámbito legislativo y judicial hasta ser considerada la guarda o custodia compartida como la primera opción, cuando este régimen resulte el más razonable y eficiente para el desarrollo integral de los hijos (Zaidán, 2016).

De ahí que, el fundamento y la naturaleza de la tenencia compartida como una nueva forma jurídica de ejercicio de la autoridad parental, puede ser encontrado a través de los sujetos que intervienen en ella, en el derecho que poseen los hijos de conservar su vínculo de hecho con sus progenitores, pero también, en el principio constitucional de la corresponsabilidad de ambos en sus derechos y obligaciones respecto de la debida atención que deben prestar a las necesidad de sus hijos objetos de ese beneficio, independientemente de la situación en la que se encuentren.

Refiriéndose particularmente a la posibilidad de llevar a cabo sus funciones de modo equitativo y bajo categorías de igualdad de trato y prohibición de todo tipo discriminación, sobre la base de su entendimiento en favor, no solo, de los situaciones materiales indispensables para la manutención de su progenie, sino también de los momentos de su desarrollo emocional, psicológico, social e intelectual, como actos que transcienden la titularidad de la patria potestad y ponen de manifiesto el principio del interés superior de los niños, niñas y adolescentes, que conforme lo señalado por la Corte Constitución del Ecuador debe ser entendido como:

(...) un principio cardinal en materia de derechos de los niños, niñas y adolescentes, que tiene una consideración primordial al momento de la adopción de todo tipo de medidas, en los ámbitos públicos y privados, que 
les conciernen, ya que goza incluso de reconocimiento internacional universal y, a través del tiempo, adquirió el carácter de norma de derecho internacional. (Sentencia Nro. 064-15-SEP-CC, 2015, p. 20)

Se trata pues, de un modalidad de tenencia que frente a la posibilidad de ejercicio conjunto y sin distinciones de la guarda y la custodia, sin lugar a dudas posee indudables beneficios, al facilitar a los hijos menores de edad la posibilidad de disfrutar de la presencia de ambos progenitores y al evitar que tengan sentimientos negativos sobre la posibilidad de su abandono, entre otras emociones que a nivel emocional y psicológico, les puede generar una eventual separación, constituyéndose en una prioridad al ayudarles a que pese al rompimiento afectivo de los progenitores se conserve un cuadro familiar de referencia que sostenga un desarrollo armónico de su personalidad y evite el llamado Síndrome de Alienación Parental (Espinoza, 2018).

Pero que también, a pesar de brindar excelentes beneficios conserva ciertos detractores que la consideran favorable únicamente en ciertos casos de plena cooperación entre los progenitores, frente al impacto negativo que puede generar en el desarrollo de los menores, ciertas situaciones de conflictividad como el hecho de alejarlos de la rutina necesaria para la edad en la que viven o la exposición a la que se pueden ver enfrentados al no permanecer regularmente con su madre y tener que convivir cada cierto lapso con el padre, en un constante e inestable peregrinaje, con las posibles afectaciones físicas que esta circunstancia podría generar en su estado emocional.

No obstante, lo que estas personas olvidan es que la tenencia compartida no solo es compatible con un escenario ideal de moderada conflictividad entre los padres que en varias ocasiones se ha intentado equiparar con el modelo estable de comunicación obligatorio para una buena tenencia compartida, o que los grados de conflictividad no difieren de otros supuestos como la tenencia individual o el régimen de visitas por muy amplio que sea, sino que también, esta institución puede ser factible y funcional aunque las partes no cooperen, siempre que cada uno acepte su posición diferenciada y tenga en consideración las necesidades del menor, en base a normas claras, en donde exista 
coherencia en la forma disciplinarlos y educarlos, como fundamento eficaz de sus distintas modalidades.

De ahí que, la procedencia de convenir o disponer un régimen de estas particularidades debe ser valorado en cada caso particular, sin que sea indispensable una facultad legal para que pueda establecerse, al encontrarse incorporada en la propia relación parental anterior a la ruptura, sin embargo, tal como manifiesta Ibañez (2004) las diversas situaciones que pueden generarse en su implementación hacen necesario que sus efectos deban ser complementados mediante un concepto que integre sus diversos contenidos en una interpretación única que fundamentada en: (...) una distribución equilibrada de las responsabilidades y de los tiempos de convivencia de los hijos con cada uno de sus padres, a ser posible consensuada entre ellos" (p. 13), así como, en la disminución de la evocación de vencedor y vencido dentro de un proceso judicial, permita a los progenitores interactuar con grados alternos de decisión en sus responsabilidades parentales conforme el tipo de relación que deseen conservar desde ese instante.

\section{Análisis legal de la tenencia compartida en el Ecuador}

Una vez analizado el tema desde el punto de vista de la doctrina, resulta transcendental revisar su regulación positiva en el ordenamiento jurídico ecuatoriano como fundamento de la institución. En este sentido, debemos comenzar manifestando que hasta antes de la expedición de la Ley Reformatoria al Código Civil (2015) publicada en el Registro Oficial Suplemento Nro. 256, de fecha 19 de junio del 2015, la única ley que regulaba la guarda y la custodia en nuestro país era el Código Civil (2005) que en su artículo 108 a pesar de limitarla a una cuestión exclusiva de los casos de disolución de la sociedad conyugal, la establecía de la siguiente manera:

En la misma audiencia, los cónyuges o sus procuradores especiales acordarán la situación económica en la que deben quedar los hijos menores de edad después de la disolución del matrimonio, la forma como deben proveer a la protección personal, educación y sostenimiento de aquéllos. Los hijos deberán estar representados por uno o más curadores 
ad - liten, según el caso, cuya designación la hará el juez prefiriendo, en lo posible, a los parientes cercanos de los hijos. (p. 33)

No obstante, esta disposición si bien buscaba que los padres procuraran llegar a un acuerdo sobre la situación económica y la protección personal de los hijos menores de edad, donde primara especialmente su interés superior, y previa audiencia en los casos de los hijos púberes, -a pesar de que la última decisión siempre la tenía el juzgador-, la verdad de los hechos es que este pacto estaba pensado desde un modelo de tenencia monoparental parcial y exclusivo, en la medida que una vez disuelto el vínculo matrimonial la regla general era que la guarda y la custodia se otorgaba exclusivamente en favor de uno de los progenitores que habitualmente era la madre, a quien además se le adjudicaba la vivienda familiar en desmedro del padre que solamente tenía el derecho de visita y la obligación legal de suministrar una pensión de alimentos en favor de sus hijos.

De esta manera, la regulación legal que establecía el Código Civil, partía de un criterio individual de atribución de la custodia, por lo que, si analizamos las normas de esta codificación, podemos deducir que solo a quien se le confería la custodia exclusiva de los hijos, terminaba a su vez siendo la persona que tenía la potestad de tomar todas aquellas decisiones relativas a la vida diaria de los menores, a pesar de que el Código Civil al no tener una regulación expresa sobre el régimen de la tenencia compartida, tampoco descartaba su posibilidad de aplicación de forma consensuada por las partes en el acuerdo de divorcio, la cual en la práctica tenía lugar en ocasiones muy limitadas como por ejemplo en la mismas facultad individual de la custodia donde podía observarse una custodia compartida de hecho o de facto debido a la extensión del régimen de visitas asignada al otro cónyuge (Monterroso y Rodríguez, 2011).

Dicho precepto estaría vigente hasta la reforma del 22 de mayo del 2015, incorporada por el Código Orgánico General de Procesos, que ante una clara preferencia de la figura materna, eliminó su contenido y modificó las normas del cuidado y crianza de los hijos menores o incapaces de cualquier edad, por la regulación contemplada en el 
Código de la Niñez y Adolescencia, que si bien en aplicación del principio de igualdad de trato y la prohibición de discriminación contemplado en el artículo 11, numeral 2 de la Constitución de la República, hace una especial referencia al desarrollo integral del hijo o hija de familia, sin variación del ejercicio conjunto de la patria potestad, mantendrá ante la separación de la pareja, rezagos de una idea tradicional de tenencia monoparental o exclusiva determinada por la entrega de los hijos menores de edad a uno de los progenitores.

Deduciéndose de la redacción del referido artículo una evidente contradicción respecto de los derechos y obligaciones de los padres en lo referente a la tenencia de sus hijos de forma solidaria o conjunta, en la medida que por una parte se confía el cuidado y crianza a uno de los progenitores y a línea seguida se hace referencia a un ejercicio conjunto de la patria potestad, que en la cotidianidad lo único que generó son diversas disputas entre estos, como consecuencia de su inadecuada utilización a manera de instrumento de venganza o con el propósito de que a uno de ellos se le establezca la custodia permanente.

Resultado evidente ante esta inseguridad jurídica de los derechos parentales en una cultura que ha retirado al menor como eje de su interés, dentro de un contexto de sociedades con tendencias igualitarias, la necesidad de generar una reforma legal que concentre las diferentes reivindicaciones que sobre esta institución se han desarrollado en las sociedades actuales, pero que también tome en cuenta los derechos consagrados en la Constitución de la República, que al considerar a los niños, niñas y adolescentes como sujetos de derechos en lugar de objetos de tutela y protección, ha generado la necesidad de evolucionar a conceptos como la guarda y la tenencia compartida que permitan a los padres que pasen por un proceso de divorcio estar presentes simultáneamente en todas las etapas y actividades que desarrollen sus hijos menores de edad de acuerdo a las mismas condiciones y derechos sobre ellos (Badaraco, 2018). 
Así, la figura de la tenencia compartida aparece como una respuesta a una realidad actual de los grupos familiares de nuestro tiempo, pues, así como se han modificado las funciones y roles de la familia dentro del matrimonio, el reconocimiento de la existencia de diversos tipos de hogares que desbordan lo estrictamente biológico y han dado paso a una noción de familia diversa adecuada a los contextos socio-culturales (Pliego, 2013), que comprende las familias: transnacionales, nucleares, sin hijos, adoptiva, de padres o madres divorciados o separados, “(...) familias ensambladas que se conforman cuando uno de los cónyuges o compañeros trae a sus hijos previos a la nueva unión, familia de padres y madres de distintas etnias o culturas, la familia homoparental, familia de crianza, entre otras" (Bolaños y Charry, 2018, p. 405), ha generado los mismos cambios respecto del cuidado de los hijos, pues en la actualidad dado que la mujer también contribuye con su trabajo y fuera de él, no se puede pensar en una custodia monoparental femenina sino de ambos progenitores.

De ahí, la necesidad de desarrollar una verdadera y auténtica tenencia compartida, que, partiendo de la idea de la plena igualdad jurídica de derechos y obligaciones entre los progenitores, así como, de la corresponsabilidad parental a pesar del rompimiento de la pareja, permita la continuación del ejercicio equitativo de la patria potestad en favor de ambos progenitores, no solo, en los casos de acuerdo sino también como primera opción del juez al instante de resolver, cuando fuera solicitada por uno de ellos, ya que solo mediante un régimen de esta naturaleza se puede dividir de modo igualitario las cargas parentales y se puede hacer efectivo el derecho al cuidado de los hijos, mediante la creación de una ficción jurídica que procura la conservación de una modalidad familiar que realmente se ha perdido debido a que estos ya no viven juntos.

En este punto, cabe señalar que, como respuesta para tratar de reducir esta problemática, la regulación ordinaria sobre tenencia y patria potestad, atraviesa en la actualidad un cambio de orientación, a través de la tramitación de un proyecto de Ley Orgánica Interpretativa de los artículos 106 y 118 del Código de la Niñez y Adolescencia, que busca ratificar la existencia en el Ecuador de la institución de la 
tenencia compartida por convenio de los progenitores y no por resolución judicial, considerando que al interior del territorio nacional se han exteriorizado diversos fallos de la Corte Constitucional del Ecuador, en los cuales, los jueces hacen referencia a esta figura, tales como la (Sentencia No. 021-11-SEP-CC, 2011), (Sentencia No. 064-15SEP-CC, 2015), (Sentencia No. 150-16-SEP-CC, 2016) y (Sentencia No. 288-16-SEPCC, 2016), que han sido sintetizadas dentro del proyecto presentado en la Asamblea Nacional.

Finalmente, a manera de conclusión podemos señalar que la importancia de participar en la educación y crianza sobre los hijos, es mucho más que una elección sobre el lugar de residencia, puesto que la examinada necesidad de estabilidad de un menor, debe ser determinada más en términos relacionados con las costumbres, valores y hábitos que en lapsos temporales o geográficos, con fundamento en la igualdad de derechos y oportunidades de los progenitores, que irradia a las relaciones familiares (Lepin, 2014), pero también en consideración a los derechos referentes con el desarrollo familiar, el interés superior de los niños, niñas y adolescentes, la dignidad de las personas, así como, los parámetros y lineamientos legales sobre los cuales se deberá reconocer la tenencia compartida en el Ecuador a fin de regular su extensión y complejidad.

\section{Tenencia compartida a partir del interés superior de los niños, niñas y adolescentes}

Una vez que se han desarrollado los principales temas referentes a la tenencia compartida, cabe examinar su vinculación con el interés superior de la niñez y la adolescencia, que entendido como un principio y mandato para todas las entidades estatales, vinculado al pleno ejercicio de los derechos que deben ser garantizados a los niños en sus diferentes fases de desarrollo (Ávila, 2012), claramente se encuentra consagrado en el segundo principio de la Declaración de los Derechos del Niño, en el siguiente texto:

El niño gozará de una protección especial y dispondrá de oportunidades y servicios, dispensado todo ello por la ley y por otros medios, para que 
pueda desarrollarse física, mental, moral, espiritual y socialmente en forma saludable y normal, así como en condiciones de libertad y dignidad. Al promulgar leyes con este fin, la consideración fundamental a que se atenderá será el interés superior del niño. (Asamblea General de las Naciones Unidas, 1959, p. 2)

Acorde con este precepto, lo que se conoce como interés superior sería incorporado en el ordenamiento jurídico ecuatoriano en el artículo 44 de la Constitución de la República que en relación a la obligación del Estado, la sociedad y la familia de promover de forma prioritaria el desarrollo integral de las niñas, niños y adolescentes, y asegurar el ejercicio de sus derechos, expresamente establece la necesidad de tener en cuenta el principio de su interés superior y la prevalencia de sus derechos (Asamblea Nacional Constituyente del Ecuador, 2008), por lo que, el espíritu cautelar de la norma constitucional aparecerá también establecido en el artículo 11 del Código de la Niñez y Adolescencia que lo conceptualiza como un principio orientado:

(...) a satisfacer el ejercicio efectivo del conjunto de los derechos de los niños, niñas y adolescentes; e impone a todas las autoridades administrativas y judiciales y a las instituciones públicas y privadas, el deber de ajustar sus decisiones y acciones para su cumplimiento. Para apreciar el interés superior se considerará la necesidad de mantener un justo equilibrio entre los derechos y deberes de niños, niñas y adolescentes, en la forma que mejor convenga a la realización de sus derechos y garantías. Este principio prevalece sobre el principio de diversidad étnica y cultural. El interés superior del niño es un principio de interpretación de la presente Ley. Nadie podrá invocarlo contra norma expresa y sin escuchar previamente la opinión del niño, niña o adolescente involucrado, que esté en condiciones de expresarla. (Congreso Nacional del Ecuador, 2003, p. 3)

Todo lo cual parece confirmar que, más allá del alcance conceptual y la protección constitucional del interés superior, es incuestionable que esta categoría se encuentra asociada a la problemática de estudio y, en consecuencia, cuando se habla de tenencia compartida se vuelve imperativo abordar este principio regulado en el artículo 44 de la Constitución, con la finalidad de evitar que la resolución por medio de la cual el juez aprueba un régimen de tenencia en caso de separación de los padres, no parta de un 
asunto discrecional sino de una cuestión que tomando en cuenta los justos y legítimos intereses de los progenitores se antepongan los derechos de bienestar, integridad y dignidad del menor derivados del principio del interés superior como regla que rige las acciones de los operadores públicos en asuntos de infancia y adolescencia.

De ahí que, ante la falta de una legislación que prevea la posibilidad de que los padres puedan ejercitar de forma conjunta o indistinta la titularidad del cuidado personal y la patria potestad de sus hijos, cualquier reforma que reflexionen los legisladores deberá considerar que el examen que realicen las autoridades competentes para decidir sobre una tenencia, sea ejecutado a través de un análisis que respetando el interés superior de la niñez, tenga en cuenta las cualidades de los padres, dejando de lado consideraciones basadas en estereotipos o prejuicios sociales sobre la idoneidad y capacidad parental o la preferencia materna, ya que disposiciones como las de los artículos 106 y 118 del Código de la Niñez y Adolescencia que optan por la madre como titular de la tenencia, sin consideración del interés superior del niño claramente otorgan ventajas sobre el padre, pues al verse involucradas en un problema que la norma jurídica decide a su favor posiblemente no se facilitarán un acuerdo (Barros, 2013).

Todas estas circunstancias sumadas a la imposibilidad de que los padres actuando de común acuerdo puedan determinar que el ejercicio del cuidado personal sea realizado de forma conjunta, fundamentan la necesidad de generar una reforma legal sobre el régimen de la tenencia que respetando los derechos a la igualdad de trato y la prohibición de discriminación determinados en el artículo 11, numeral 2 de la Constitución, en el caso de que ambos padres demuestres iguales condiciones, incorpore la posibilidad de que los operadores de justicia puedan escoger como primera opción confiar la tenencia a los dos, siempre que al ser solicitada por una de las partes se tenga en cuenta lo que más convenga a los intereses de los menores antes que a las motivaciones o razones de los padres, pues al concederles los mismos derechos se reduce la posibilidad de una disputa por el control del escenario posterior al divorcio y los beneficios económicos (Catalán, 2011). 
A partir del análisis efectuado, se presenta un resumen de la estructura investigativa desarrollada referente a la problemática de la tenencia compartida en el Ecuador a partir del interés superior de los niños, niñas y adolescentes, en la siguiente figura:

\section{ANÁLISIS DE LA TENENCIA COMPARTIDA EN EL ECUADOR A PARTIR DEL INTERÉS SUPERIOR DE LOS NIÑOS, NIÑAS Y ADOLESCENTES}

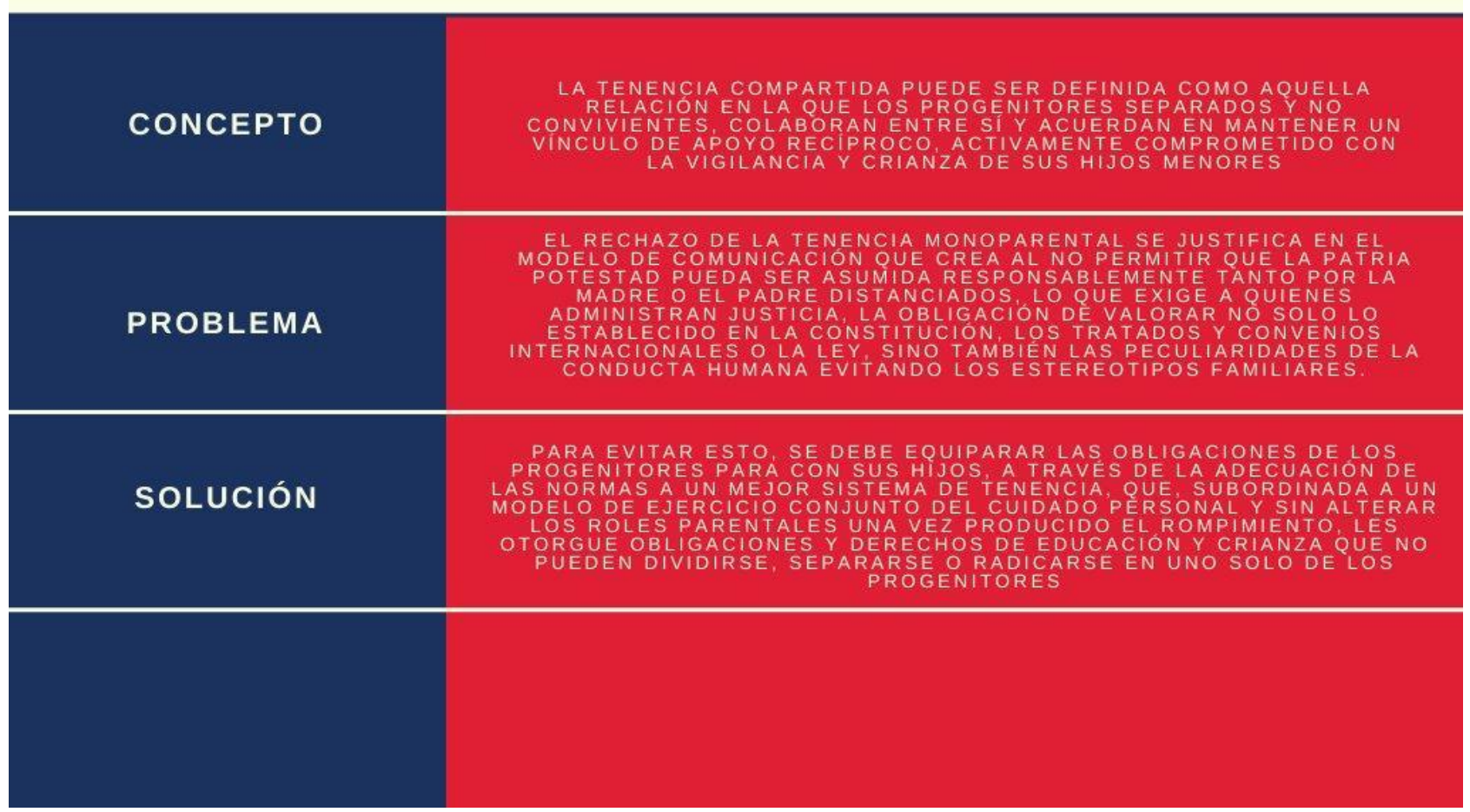

Figura 1: Análisis de la tenencia compartida en el Ecuador a partir del interés superior de los niños, niñas y adolescentes 


\section{METODOLOGÍA}

Por tratarse de una investigación teórico-exploratoria, la metodología empleada para llevar a cabo el presente trabajo referente a la tenencia compartida se motivó en un paradigma cualitativo, que, partiendo de un análisis crítico de la información jurídica se ha convertido en un instrumento adecuado para el análisis de los contenidos normativos y axiológicos del sistema actual de tenencia regulado en el Ecuador, así como, de las implicaciones que generan la falta de una legislación subordinada a un modelo de ejercicio conjunto del cuidado personal mediante el análisis de sus características (Almeida, Erazo, Ormaza y Narváez, 2020).

Es así que, la investigación se situó en el método analítico-sintético que al ubicar el tema de estudio dentro de un marco teórico conceptual ha permitido la determinación de los aspectos básicos del problema desde la descomposición de su objeto a cada una de sus particularidades, con la finalidad de examinarlas en forma individual para integrarlas a un examen exhaustivo de su naturaleza, causas y conexiones que origina la falta de una regulación de la tenencia compartida en el Ecuador (Behar, 2008).

Así mismo, este trabajo se realizó en el ámbito del método inductivo-deductivo, que articulado al estudio del régimen de la tenencia compartida a partir de sus premisas particulares ha permitido mediante la observación, el registro de datos, la examinación y la derivación inductiva el establecimiento de regularidades y conclusiones generales aplicables a casos semejantes (Gómez, 2012).

\section{APORTES GENERADOS}

En el presente trabajo se exponen las principales características del régimen de tenencia regulado en el estado ecuatoriano, puesto que la disolución del grupo familiar por diferentes motivos como el divorcio, la separación, la nulidad, las discrepancias u otras causas, bajo ciertas condiciones devienen en hijos expuestos a múltiples factores de riesgo que ocasionalmente pueden convertirlos en objeto de chantaje, maltrato o incluso de triunfo frente a las disputas de padres irresponsables, que ven en su custodia 
un mecanismo para forzar la presencia de uno de los progenitores, garantizar la manutención o crear desequilibrio en la pareja frente a nuevos planes familiares.

Esto se ve agravado debido a la existencia de normas jurídicas como las contenidas en los artículos 106 y 118 del Código de la Niñez y Adolescencia, que si bien es innegable buscan el bienestar y la integridad de los menores de edad, al no prever la posibilidad de que los padres puedan ejercitar de forma conjunta o indistinta la titularidad del cuidado personal y la patria potestad, en diferentes ocasiones han generado que las resoluciones de los operadores de justicia deriven en resultados adversos al interés superior, al impedir a los progenitores que ante la falta de acuerdo y frente a la preferencia materna puedan ejercerse de forma conjunta los deberes y derechos de criar y educar, vinculados con una mayor capacidad de decisión sobre los hijos de padres separados.

Es por esta razón que, bajo la legislación actual frente a la alteración de las funciones parentales que desfiguran la familia, la motivación para el rechazo de la tenencia monoparental es legítima inclusive para quienes aparentemente se ven favorecidos de esta forma unilateral de atribución, por cuanto, el modelo de comunicación creado no permite que la patria potestad pueda ser asumida responsablemente tanto por la madre o el padre distanciados, pues en muchos casos el peso de la crianza de los hijos cae únicamente sobre las espaldas de uno de los progenitor creando un entorno familiar de inestabilidad, que exige a quienes administran justicia, su obligación de valorar no solo lo establecido en la Constitución, los tratados y convenios internacionales o la ley, sino también las peculiaridades de la conducta humana evitando los estereotipos familiares. Para esto, se deberán equiparar las obligaciones de los progenitores para con sus hijos, a través de la adecuación de las normas a un mejor sistema de tenencia, que, subordinada a un modelo de ejercicio conjunto del cuidado personal y sin alterar los roles parentales una vez producido el rompimiento, les otorgue atribuciones que tradicionalmente no ocupaban por múltiples factores. Un sistema de esta naturaleza capaz de generar una igualdad en los derechos de los padres, permitirá reducir los 
conflictos de la pareja, tanto en el cuidado personal como en la determinación del pago de la pensión de alimentos, ya que las obligaciones y derechos de educación y crianza son en principio inalienables y no pueden, por tanto, dividirse, separarse o radicarse en uno de los progenitores.

\section{REFERENCIAS CONSULTADAS}

1. Almeida Toral, P. F., Erazo Álvarez, J. C., Ormaza Ávila, D. A., \& Narváez Zurita, C. I. (2020). La aplicación de los derechos humanos en el interés superior del niño. Iustitia Socialis, 639. doi:http://dx.doi.org/10.35381/racji.v5i8.594

2. Asamblea General de las Naciones Unidas. (1959). Declaración de los Derechos del Niño.

3. Asamblea Nacional Constituyente del Ecuador. (2008). Constitución de la República del Ecuador. Ciudad Alfaro Montecristi, Manabí, Ecuador: Registro Oficial Nro. 449.

4. Asamblea Nacional República del Ecuador. (2015). Ley Reformatoria al Código Civil. Quito, Pichincha, Ecuador: Registro Oficial Nro. 526.

5. Ávila Santamaría, R. (2012). Los derechos y sus garantías. Ensayos críticos. Quito: Centro de Estudios y Difusión del Derecho Constitucional (CEDEC).

6. Badaraco Delgado, V. (2018). La tenencia compartida en el Ecuador ¿Una necesidad? Espirales revista multidisciplinaria de investigación, 31-39.

7. Barros Aravena, F. (2013). El cuidado personal, igualdad entre padres e interés superior del niño. Tesis para obtar al grado de magíster en Derecho. Universidad de Chile.

8. Behar Rivero, D. (2008). Metodología de la Investigación. Shalom.

9. Bolaños Enríquez, T., \& Charry Morales, A. (2018). Prejuicios y homosexualidad, el largo camino hacia la adopción homoparental, especial atención al caso colombiano. Estudios Constitucionales, 395-424.

10. Cajamarca, K. I. (2016). La tenencia compartida: solución o conflicto. Cuenca: Universidad de Cuenca. 
11. Catalán Frías, M. (2011). La custodía compartida. Revista de Derecho y Criminología, 57-82.

12. Congreso Nacional del Ecuador. (2003). Código de la niñez y adolescencia. Quito, Pichincha, Ecuador.

13. Congreso Nacional del Ecuador. (2005). Código Civil. Quito, Pichincha, Ecuador: Registro Oficial Nro. 46.

14. Espinoza de los Monteros, R. Z. (2018). Nadie pierde: La guarda y custodia compartida. Aspectos jurídicos-procesales. Madrid: DYKINSON.

15. Gómez Bastar, S. (2012). Metodología de la investigación. México: RED TERCER MILENIO S.C.

16. Ibañez Valverde, V. (2004). El laberinto de la custodia compartida. Claroscuros de un solo nombre con varios significados. Boletin de Derecho de Familia, 1-15.

17. Lepin Molina, C. (2014). Los nuevos principios del Derecho de Familia. Revista Chilena de Derecho Privado, 9-55.

18. Monterroso Casado, E., \& Rodríguez de Almeida, M. (2011). Análisis de la regulación legal de la custodía compartida tras la separación y el divorcio: una propuesta de lege ferenda. Revista CEFLEGAL Nro. 131, 5-58.

19. Morales Ortega, H., \& Castillo Bolaño, J. (2011). La custodia compartida: un análisis desde la perpectiva de género y de derecho. Justicia, 56-70.

20. Pliego Carrasco, F. (2013). Tipos de familia y bienestar de niños y adultos. El debate cultural del siglo XXI en 13 países democráticos (Primera ed.). México: Instituto de Investigaciones Sociales de la Universidad Nacional Autónoma de México.

21. Sentencia No. 021-11-SEP-CC, Causa No. 0317-09-EP (Corte Constitucional del Ecuador 01 de Septiembre de 2011).

22. Sentencia No. 064-15-SEP-CC, Causa No. 0331-12-EP (Corte Constitucional del Ecuador 11 de Marzo de 2015).

23. Sentencia No. 150-16-SEP-CC, Causa No. 1201-14-EP (Corte Constitucional del Ecuador 4 de Mayo de 2016).

24. Sentencia No. 288-16-SEP-CC, Causa No. 0702-10-EP (Corte Constitucional del Ecuador 7 de Septiembre de 2016). 
25. Sentencia Nro. 064-15-SEP-CC, Caso Nro. 0331-12-EP (Corte Constitucional del Ecuador 11 de Marzo de 2015).

26. Tamayo Vásquez, F., \& Pino Loza, E. (2019). Derechos Humanos de los niños, niñas y adolescentes en los procesos Arbitrales. IUSTITIA SOCIALIS, 4(7), 97114. doi:http://dx.doi.org/10.35381/racji.v4i7.357

27.Zabala, H. R. (2014). El Divorcio o Separación Encaminada a la Tenencia Compartida de los Padres. Quito: Universidad Central del Ecuador.

28.Zaidán Albuja, S. (2016). El derecho constitucional de cuidado de los hijos: normativa. Programa de Maestría Profesional en Derecho Constitucional. Quito, Pichincha, Ecuador: Universidad Andina Simón Bolívar .

(C2020 por los autores. Este artículo es de acceso abierto y distribuido según los términos y condiciones de la licencia Creative Commons Atribución-NoComercial-Compartirlgual 4.0 Internacional (CC BY-NC-SA 4.0) (https://creativecommons.org/licenses/by-nc-sa/4.0/). 